\title{
BMJ Open Predictors of in-hospital mortality among patients with pulmonary tuberculosis: a protocol of systematic review and meta-analysis of observational studies
}

\author{
Carlos Podalirio Borges de Almeida, ${ }^{1}$ Rachel Couban, ${ }^{2}$ Sun Makosso Kallyth, ${ }^{3}$ \\ Vagner Kunz Cabral, ${ }^{1}$ Samantha Craigie, ${ }^{2}$ Jason Walter Busse, ${ }^{4,5}$ \\ Denise Rossato Silva ${ }^{1,6}$
}

To cite: Almeida CPBde, Couban R, Kallyth SM, et al. Predictors of in-hospital mortality among patients with pulmonary tuberculosis: a protocol of systematic review and meta-analysis of observational studies. BMJ Open 2016;6:e011957. doi:10.1136/bmjopen-2016011957

- Prepublication history and additional material is available. To view please visit the journal (http://dx.doi.org/ 10.1136/bmjopen-2016011957).

Received 18 March 2016 Revised 14 June 2016 Accepted 5 August 2016

CrossMark

For numbered affiliations see end of article.

\section{Correspondence to}

Dr Carlos Podalirio Borges de Almeida;

carlosalmeida1410@hotmail. com

\section{ABSTRACT}

Introduction: Tuberculosis (TB) continues to be a major public health issue worldwide, with 1.4 million deaths occurring annually. There is uncertainty regarding which factors are associated with in-hospital mortality among patients with pulmonary TB. This knowledge gap complicates efforts to identify and improve the management of those individuals with $T B$ at greatest risk of death. The aim of this systematic review and meta-analysis is to establish predictors of in-hospital mortality among patients with pulmonary TB to enhance the evidence base for public policy.

Methods and analysis: Studies will be identified by a MEDLINE, EMBASE and Global Health search. Eligible studies will be cohort and case-control studies that report predictors or risk factors for in-hospital mortality among patients with pulmonary TB and an adjusted analysis to explore factors associated with inhospital mortality. We will use the Grading of Recommendations Assessment, Development and Evaluation approach to summarise the findings of some reported predictors. Teams of 2 reviewers will screen the titles and abstracts of all citations identified in our search, independently and in duplicate, extract data, and assess scientific quality using standardised forms quality assessment and tools tailored. We will pool all factors that were assessed for an association with mortality that were reported by $>1$ study, and presented the $\mathrm{OR}$ and the associated $95 \% \mathrm{Cl}$. When studies provided the measure of association as a relative risk (RR), we will convert the $R R$ to $O R$ using the formula provided by Wang. For binary data, we will calculate a pooled OR, with an associated $95 \% \mathrm{Cl}$.

Ethics and dissemination: This study is based on published data, and therefore ethical approval is not a requirement. Findings will be disseminated through publication in peer-reviewed journals and conference presentations at relevant conferences.

Trial registration number: CRD42015025755.

\section{BACKGROUND}

In 2010, an estimated 12.0 million people worldwide were living with active pulmonary

\section{Strengths and limitations of this study}

- Our search will be performed in close cooperation with a specialised research librarian with health research methodology knowledge.

- The screening and extraction will be performed cooperatively by two researchers employing pretested, standardised extraction forms.

- The review may include novel studies from several regions and there is no restriction to any language and period.

- Our study may be easily influenced by threats to credibility (ie, internal validity) and applicability (ie, external validity).

- The study will involve judgements made by review authors, which can result in bias.

tuberculosis (TB), with 9 million new cases and 1.4 million deaths due to $\mathrm{TB}$ occurring annually. TB continues to be a major public health issue worldwide, particularly in low and middle income countries, despite rigorous efforts to contain its spread and implementation of effective treatment strategies. ${ }^{1-7}$

A variety of factors have been associated with a greater risk of death among patients with $\mathrm{TB}$, including poverty, homelessness, alcohol or drug addiction, irregular or inadequate treatment, late diagnosis of the disease, multidrug-resistant TB (MDR-TB) and advanced age. ${ }^{5}$ Furthermore, HIV infection is an important factor related to the increased morbidity and mortality of TB in different world regions, and has resulted in an increased number of hospital admissions due to $\mathrm{TB}^{48}$

Even in developed countries where the overall incidence of $\mathrm{TB}$ is low, it remains common among the elderly population due to prolonged life expectancy, use of drugs that 
suppress cellular immunity, and delay of the diagnosis of TB in the aged. ${ }^{4}$ TB does not usually require hospital admission for treatment, but if symptoms such as shortness of breath and deterioration in a systemic condition are present, hospitalisation may be necessary. A large proportion of patients with TB are hospitalised, and estimates of in-hospital mortality range from $2 \%$ to $12 \% .^{8-12}$ Despite the recommended outpatient care, most of the current costs of TB treatment result from hospitalisation. ${ }^{13}$

Some cases may need to be treated at the intensive care unit (ICU), such as cases of acute respiratory failure due to TB. The cases of TB requiring intensive care represent $1-3 \%$ of all patients with TB. The most common reasons for ICU admission of patients with TB are the development of acute respiratory distress syndrome and severe organ failure, such as renal failure. Besides, the mortality rate of TB patients requiring intensive care due to acute respiratory failure has been reported to be approximately $60 \% .^{1415}$

Patients with TB staying in-hospital have a higher risk of mortality in comparison with patients with TB receiving treatment in other health services, like primary care and ambulatory care services. TB deaths are crucial indicators in TB programme monitoring, ${ }^{8-12}$ especially in areas with high HIV and TB prevalence. Data on TB deaths provide us with a better understanding of the causes of these deaths and help guide interventions to reduce mortality. Considering that there is uncertainty regarding which factors are associated with in-hospital mortality among patients with pulmonary $\mathrm{TB}$, and that a large proportion of patients with TB are hospitalised, it is important to fill this knowledge gap to identify and improve the management of those individuals with TB at greatest risk of death. The aim of this systematic review and meta-analysis is to establish predictors of in-hospital mortality among patients with pulmonary TB to enhance the evidence base for public policy.

\section{METHODS}

\section{Search strategy}

We will use a multimodal search strategy focused on three bibliographical databases (MEDLINE, EMBASE and Global Health), personal files, consultation with experts and review of bibliographies among eligible articles. An experienced librarian (RC) will use Medical Subject Headings, adding terms and keywords from a preliminary search to develop the database search strategies. In each database, the librarian will use an iterative process to refine the search strategy through testing several search terms and incorporating new search terms as new relevant citations will be identified. The search will include the following databases from inception to November 2015: MEDLINE, EMBASE and Global Health. The search will consist of three concepts combined using AND operator. The first concept is TB, the second is hospitalisation and the third is mortality (see online supplementary appendix 1 ).

\section{Study selection}

\section{Eligibility criteria}

Eligible trials will meet the following criteria: (1) the study is an observational study (cohort or case-control studies); (2) the study reported predictors or risk factors for in-hospital mortality among patients with pulmonary TB and (3) the authors report an adjusted analysis to explore factors associated with in-hospital mortality.

The main outcome will be death as defined by the WHO, author's judgement or medical records.

\section{Assessment of study eligibility}

Teams of two reviewers trained in health research methodology will screen the titles and abstracts of all citations identified in our search, independently and in duplicate, and if either reviewer thought that a citation might be eligible, we retrieved the study for full-text review. Disagreements will be resolved by consensus, with consultation of a third investigator when resolution could not be achieved. We will measure agreement between reviewers to assess the reliability of full-text review using the guidelines proposed by Landis and Koch. ${ }^{16}$ Precisely, we will use the kappa statistic, and interpret them using the following thresholds: $<0.20$ as slight agreement, $0.21-0.40$ as fair agreement, $0.41-0.60$ as moderate agreement, $0.61-0.80$ as substantial agreement and $>0.80$ as almost perfect agreement.

\section{Assessment of study quality}

Pairs of reviewers assessed risk of bias, independently and in duplicate. We used the following criteria from the Users' Guides to the Medical Literature to address risk of bias: (1) representativeness of the study population (low risk of bias when using random sampling, consecutive sampling, or data collected from a national or international registry; high risk of bias when the source of study population was not reported or acquired through convenience sampling); (2) validity of outcome assessment (how the authors define mortality? Did they evaluate only TB-related deaths?) and (3) whether or not predictive models were optimally adjusted (low risk of bias if adjusted for, at minimum, age, sex and HIV status).

We will use the Grading of Recommendations Assessment, Development and Evaluation (GRADE) approach to summarise the findings of some reported predictors. ${ }^{17}$ GRADE has been adopted by over 70 organisations worldwide, and this approach facilitates transparent, rigorous and comprehensive assessment of evidence quality on a per outcome basis. ${ }^{18-22}$

We will categorise the confidence in estimates (quality of evidence) as high, moderate, low or very low. GRADE guidance will be used to determine whether to rate down confidence in the body of evidence for risk of bias $^{21}$ and for imprecision, ${ }^{18}$ inconsistency ${ }^{19}$ and publication bias. $^{20}$

When plausible worst case scenarios reverse the treatment effect, we will rate down for risk of bias. The 
results of meta-analyses will be presented in GRADE evidence profiles that will provide a succinct, easily digestible presentation of the risk of bias and magnitude of effects. ${ }^{17}$ In case of doubt or missing details, authors will be contacted for clarification.

\section{Data abstraction and analysis}

Two reviewers will extract data from each eligible study, including demographic information (eg, gender, age, race, etc), methodology and all reported predictors.

When possible, we will pool all factors that were assessed for an association with mortality that were reported by $>1$ study, and presented the OR and the associated 95\% CI. When studies provided the measure of association as a relative risk (RR), we will convert the RR to an OR using the formula provided by Wang. ${ }^{23}$ When possible, we will pool outcome data across trials. For binary data, we will calculate a pooled OR, with an associated $95 \%$ CI.

When we identified only one study addressing a given predictor in an adjusted analysis, or $\geq 2$ studies explored a given predictor but authors did not present data necessary for a pooled analysis, we will summarise the reported associations. We will explore the consistency of association between our pooled results and studies reporting the same predictors that were not possible to pool. We will use the following three criteria to identify predictors not included in the pooled analyses that showed promise for future research: (1) a statistically significant association with mortality of $\mathrm{p} \leq 0.01$; (2) a large magnitude of association ( $\mathrm{OR} \geq 2.0)$ and (3) a sample size of $\geq 200$.

Authors creating predictive models may choose to enter independent variables into an adjusted analysis only if they meet a threshold for statistical significance in a bivariable analysis. Further, some authors do not report the associated data for predictors that were not significant in their adjusted analysis. Thus, there is a risk of overestimating the strength of association by restricting statistical pooling to predictors that appear in adjusted regression models and for which data are provided. To address this risk, we imputed an OR of ' 1 ' for predictors that were tested in bivariable analyses but because of non-significance excluded from adjusted analyses, or included in multivariable analyses with the only information provided being that they were 'not significant'. We imputed an associated variance for all such predictors using the hot-deck approach. ${ }^{24}$

The $\mathrm{I}^{2}$ statistic, the percentage of between-study variability that is due to true differences between studies (heterogeneity) rather than sampling error (chance), will be used to quantify inconsistency among studies. ${ }^{25-27}$ Values of $30-60 \%$ may represent moderate heterogeneity, 50-90\% substantial heterogeneity, and $75-100 \%$ considerable heterogeneity. ${ }^{26}{ }^{27}$ The random effect meta-analysis model will be used on the pooled data through the inverse-variance random effects method. The software STATA will be used.
If we find heterogeneity, we will perform subgroup analysis to understand and explain the source of the heterogeneity. We will conduct a test of interaction and, if significant, we will report the results separately for each subgroup. Meta-regression and subgroup analyses will be performed to explore and interpret the results in the context of the GRADE system. ${ }^{28}$

We have generated five a priori hypotheses to explain variability between studies:

1. Patients at an ICU will have higher mortality than patients in other places at hospital.

2. Pulmonary TB in patients with HIV will be associated with a lower survival rate in comparison with patients without HIV.

3. Patients with comorbidity (eg, diabetes mellitus, chronic renal diseases, cancer, HIV, chronic use of immunosuppressive drugs) will show a lower survival rate versus patients without comorbidity.

4. Trials with a small sample size will $(<100)$ show higher mortality among patients with pulmonary $\mathrm{TB}$ than trials with a bigger sample size $(>100)$.

5. Trials with a higher risk of bias will demonstrate higher mortality rates than trials with a lower risk of bias.

Sensitivity analysis will be performed to determine any bias introduced by the eligibility criteria, analysed data, analysis method and other relevant issues identified during the review process. Publication bias will be assessed using funnel plots for the included studies. ${ }^{29}$

\section{Ethics and dissemination}

This study is based on published data, and therefore ethical approval is not a requirement. This systematic review and meta-analysis is expected to serve as a basis for designing preventive and control strategies for in-hospital patients with $\mathrm{TB}$, and as a guide for future research based on the remaining gaps. It is anticipated that findings from the review will be useful for informing policy, practice and research priorities for improving the management of in-hospital patients with TB. Findings will be disseminated through publication in peer-reviewed journals and conference presentations at relevant conferences. We also plan to update the review in the future to monitor changes and guide health services and policy solutions.

\section{Author affiliations}

${ }^{1}$ Respiratory Sciences Program, Universidade Federal do Rio Grande do Sul, Porto Alegre, Hospital de Clínicas de Porto Alegre, Porto Alegre, Rio Grande do Sul, Brazil

${ }^{2}$ The Michael G. DeGroote Institute for Pain Research and Care, McMaster University, Hamilton, Ontario, Canada

${ }^{3}$ The Michael G. DeGroote Institute for Pain Research and Care, McMaster University, Hamilton, Ontario, Canada

${ }^{4}$ Department of Anesthesia, McMaster University, Hamilton, Ontario, Canada

${ }^{5}$ Department of Clinical Epidemiology \& Biostatistics, The Michael

G. DeGroote Institute for Pain Research and Care, McMaster University,

Hamilton, Ontario, Canada

${ }^{6}$ Faculty of Medicine, Pulmonology Division, Universidade Federal do Rio

Grande do Sul; Hospital de Clínicas de Porto Alegre, Porto Alegre, Rio Grande do Sul, Brazil 
Contributors All authors made substantial contributions to conception and design. CPBdA designed the study, collected the data and wrote the manuscript. RC designed the search strategy. SMK designed the study and analysed the data. VKC collected the data and wrote the manuscript. SC designed the study and collected the data. JWB designed the study, analysed the data and wrote the paper. DRS designed the study, collected the data, and wrote the paper, as well as revised it critically for important intellectual content. All authors provided final approval of the version to be published.

Funding Coordenação de Aperfeiçoamento de Pessoal de Nível Superior (Capes) Fundo de Incentivo à Pesquisa (FIPE)/Hospital de Clínicas de Porto Alegre.

\section{Competing interests None declared.}

Provenance and peer review Not commissioned; externally peer reviewed.

Open Access This is an Open Access article distributed in accordance with the Creative Commons Attribution Non Commercial (CC BY-NC 4.0) license, which permits others to distribute, remix, adapt, build upon this work noncommercially, and license their derivative works on different terms, provided the original work is properly cited and the use is non-commercial. See: http:// creativecommons.org/licenses/by-nc/4.0/

\section{REFERENCES}

1. WHO. World Health Organization. Tuberculosis fact sheet. 2014. Geneva. 2014. Available at: www.who.int

2. Lawn SD, Zumla Al. Tuberculosis. Lancet 2011;378:57-72.

3. Lui G, Wong RY, Li F, et al. High mortality in adults hospitalized for active tuberculosis in a low HIV prevalence setting. PLOS ONE 2014;9:e92077.

4. Lubart E, Lidgi M, Leibovitz A, et al. Mortality of patients hospitalized for active tuberculosis in Israel. Isr Med Assoc J 2007;9:870-3.

5. Silva DR, Menegotto DM, Schulz LF, et al. Factors associated with mortality in hospitalized patients with newly diagnosed tuberculosis. Lung 2010;188:33-41.

6. Haque G, Kumar A, Saifuddin F, et al. Prognostic factors in tuberculosis related mortalities in hospitalized patients. Tuberc Res Treat 2014;2014:624671.

7. Alavi-Naini R, Moghtaderi A, Metanat M, et al. Factors associated with mortality in tuberculosis patients. J Res Med Sci 2013;18:52-5.

8. Hansel NN, Merriman B, Haponik EF, et al. Hospitalizations for tuberculosis in the United States in 2000: predictors of in-hospital mortality. Chest 2004;126:1079-86.

9. Hansel NN, Wu AW, Chang B, et al. Quality of life in tuberculosis: patient and provider perspectives. Qual Life Res 2004;13:639-52.

10. Rao VK, lademarco EP, Fraser VJ, et al. The impact of comorbidity on mortality following in-hospital diagnosis of tuberculosis. Chest $1998 ; 114: 1244-52$
11. Singleton L, Turner M, Haskal R, et al. Long-term hospitalization for tuberculosis control. Experience with a medical-psychosocial inpatient unit. JAMA 1997;278:838-42.

12. Greenaway C, Menzies D, Fanning A, et al. Delay in diagnosis among hospitalized patients with active tuberculosis-predictors and outcomes. Am J Respir Crit Care Med 2002;165:927-33.

13. Kim HJ, Lee $\mathrm{CH}$, Shin $\mathrm{S}$, et al. The impact of nutritional deficit on mortality of in-patients with pulmonary tuberculosis. Int $J$ Tuberc Lung Dis 2010;14:79-85.

14. Erbes R, Oettel K, Raffenberg M, et al. Characteristics and outcome of patients with active pulmonary tuberculosis requiring intensive care. Eur Respir J 2006;27:1223-8.

15. Levy $\mathrm{H}$, Kallenbach JM, Feldman $\mathrm{C}$, et al. Acute respiratory failure in active tuberculosis. Crit Care Med 1987;15:221-5.

16. Landis JR, Koch GG. The measurement of observer agreement for categorical data. Biometrics 1977;33:159-74.

17. Atkins D, Best D, Briss PA, et al. Grading quality of evidence and strength of recommendations. BMJ 2004;328:1490.

18. Guyatt GH, Oxman AD, Kunz R, et al. GRADE guidelines 6. Rating the quality of evidence-imprecision. J Clin Epidemiol 2011;64:1283-93.

19. Guyatt GH, Oxman AD, Kunz R, et al., GRADE Working Group. GRADE guidelines: 7. Rating the quality of evidence-inconsistency. J Clin Epidemiol 2011;64:1294-302.

20. Guyatt GH, Oxman AD, Montori V, et al. GRADE guidelines: 5. Rating the quality of evidence-publication bias. J Clin Epidemiol 2011;64:1277-82.

21. Guyatt GH, Oxman AD, Vist G, et al. GRADE guidelines: 4. Rating the quality of evidence-study limitations (risk of bias). J Clin Epidemiol 2011;64:407-15.

22. Mulla SM, Buckley DN, Moulin DE, et al. Management of chronic neuropathic pain: a protocol for a multiple treatment comparison meta-analysis of randomised controlled trials. BMJ Open 2014;4 e006112.

23. Wang Z. Converting odds ratio to relative risk in cohort studies with partial data information. J Stat Softw 2013;55:1-12.

24. Gelman $\mathrm{AH}$. Chapter 25. Missing-data imputation. In: Gelman $\mathrm{AH}$ ed. Data analysis using regression and multilevel/hierarchical models. New York: Cambridge University Press, 2006:529-44.

25. Higgins JP, Thompson SG. Quantifying heterogeneity in a meta-analysis. Stat Med 2002;21:1539-58.

26. Busse JW, Jacobs CL, Swiontkowski MF, et al. Complex limb salvage or early amputation for severe lower-limb injury: a meta-analysis of observational studies. J Orthop Trauma 2007;21:70-6.

27. Higgins JP, Thompson SG, Deeks JJ, et al. Measuring inconsistency in meta-analyses. BMJ 2003;327:557-60.

28. Altman DG, Bland JM. Interaction revisited: the difference between two estimates. BMJ 2003;326:219.

29. Egger M, Davey Smith G, Schneider M, et al. Bias in meta-analysis detected by a simple, graphical test. BMJ 1997;315:629-34 